\title{
Reflections on cavitation nuclei in water
}

\author{
Mørch, Knud Aage
}

Published in:

Physics of Fluids

Link to article, DOI:

$10.1063 / 1.2747210$

Publication date:

2007

Document Version

Publisher's PDF, also known as Version of record

Link back to DTU Orbit

Citation (APA):

Mørch, K. A. (2007). Reflections on cavitation nuclei in water. Physics of Fluids, 19(7), 072104.

https://doi.org/10.1063/1.2747210

\section{General rights}

Copyright and moral rights for the publications made accessible in the public portal are retained by the authors and/or other copyright owners and it is a condition of accessing publications that users recognise and abide by the legal requirements associated with these rights.

- Users may download and print one copy of any publication from the public portal for the purpose of private study or research.

- You may not further distribute the material or use it for any profit-making activity or commercial gain

- You may freely distribute the URL identifying the publication in the public portal

If you believe that this document breaches copyright please contact us providing details, and we will remove access to the work immediately and investigate your claim. 


\title{
Reflections on cavitation nuclei in water
}

\author{
K. A. Mørch \\ Department of Physics and the Quantum Protein Center, Technical University of Denmark, \\ DK-2800 Kgs. Lyngby, Denmark
}

(Received 26 January 2007; accepted 16 May 2007; published online 10 July 2007)

\begin{abstract}
The origin of cavitation bubbles, cavitation nuclei, has been a subject of debate since the early years of cavitation research. This paper presents an analysis of a representative selection of experimental investigations of cavitation inception and the tensile strength of water. At atmospheric pressure, the possibility of stabilization of free gas bubbles by a skin has been documented, but only within a range of bubble sizes that makes them responsible for tensile strengths up to about $1.5 \mathrm{bar}$, and values reaching almost 300 bar have been measured. However, cavitation nuclei can also be harbored on the surface of particles and bounding walls. Such nuclei can be related to the full range of tensile strengths measured, when differences of experimental conditions are taken into consideration. The absence or presence of contamination on surfaces, as well as the structure of the surfaces, are central to explaining why the tensile strength of water varies so dramatically between the experiments reported. A model for calculation of the critical pressure of skin-covered free gas bubbles as well as that of interfacial gaseous nuclei covered by a skin is presented. This model is able to bridge the apparently conflicting results of the many scientists, who have been working in the field over the years. (C) 2007 American Institute of Physics. [DOI: 10.1063/1.2747210]
\end{abstract}

\section{INTRODUCTION}

The tensile stress at which a liquid ruptures into a twophase medium is named its tensile strength. By homogeneous nucleation in pure water, $\mathrm{H}_{2} \mathrm{O}$, the theoretical tensile strength is $\sim 1400$ bar at $25{ }^{\circ} \mathrm{C} .{ }^{1}$ This value has actually been achieved experimentally by isochoric cooling of ultraclean water in inclusions in quartz. ${ }^{2}$ However, experimentally the tensile strength of ordinary water is found to be very low, and it is of great importance for numerous practical applications of water. Even when great care is taken in the cleaning of normal water and equipment, the highest tensile strength measured is almost an order of magnitude smaller than the theoretical one. Therefore, some sort of cavitation nuclei has to be present. The immediate candidate would be free gas bubbles, but they either disappear by buoyancy, or shrink and dissolve due to diffusion of gas into the liquid, driven by the excess pressure in the bubble that is set up by surface tension-they are inherently unstable. ${ }^{3}$ Harvey et al. ${ }^{4}$ suggested cavitation nuclei to be gas pockets stabilized in crevices of solid surfaces, either bounding walls or particles present in the liquid. Many experimental results could be explained from their model, but not all, and other models of stabilization of free gas bubbles were developed. Thus, Fox and Herzfeld ${ }^{5}$ suggested that organic impurities such as fatty acids in the liquid form a film on the surface of free gas bubbles. When shrinking, the film was supposed to turn into a skin preventing diffusion of gas and stopping the bubbles from shrinking further, i.e., they were stabilized. Alternatively, Akulichev ${ }^{6}$ considered stabilization of free gas bubbles by assuming the bubbles to carry identical surface charges (ions), which prevented them from shrinking below some lower limit. However, experiments by Sirotyuk ${ }^{7}$ exclude this model. More recently, Yount ${ }^{8,9}$ developed a model based on a skin of variable permeability able to reduce or eliminate surface tension (the VP model). During the years, a vast amount of experimental results has been presented, often with apparently contradicting findings, and it seems relevant to make a general analysis and synthesis of at least a representative selection of these.

It is noticed that in a liquid without surface tension, a pure vapor bubble is in equilibrium only at the pressure of the vapor. Such a system has no tensile strength, and a pressure increase makes the bubble collapse by condensation of the vapor. The presence of surface tension reduces this unstable equilibrium pressure by the surface tension pressure, and thus it may be negative. An additional content of noncondensable gas in the bubble allows it to reach stable equilibrium at a bubble size that depends on the far-field pressure (though only over a limited time as the gas tends to diffuse into the liquid). However, at reduction of the pressure below a certain level, the critical pressure, the gas bubble becomes unstable and it grows explosively into a vaporous cavity.

\section{LITERATURE ANALYSIS AND SYNTHESIS}

The tensile strength of highly purified water of low gas content, strained in a "thoroughly cleaned" rotating Pyrex glass tube of inner diameter $0.6-0.8 \mathrm{~mm}$, was found by Briggs ${ }^{10}$ to come up to a maximum of about 277 bar at $\sim 10{ }^{\circ} \mathrm{C}$. The threshold dropped abruptly toward low values for temperatures approaching $0{ }^{\circ} \mathrm{C}$. "Scrupulous cleanliness" was demanded for obtaining this high tensile strength. Interestingly, in flow experiments with unfiltered tap water, the maximum of tensile strength was found by Keller ${ }^{11}$ to occur also at a temperature of $\sim 10{ }^{\circ} \mathrm{C}$, though here the absolute stress level was two orders of magnitude lower, Fig. 1. It indicates a similarity of the basic mechanisms of inception in the two cases. 


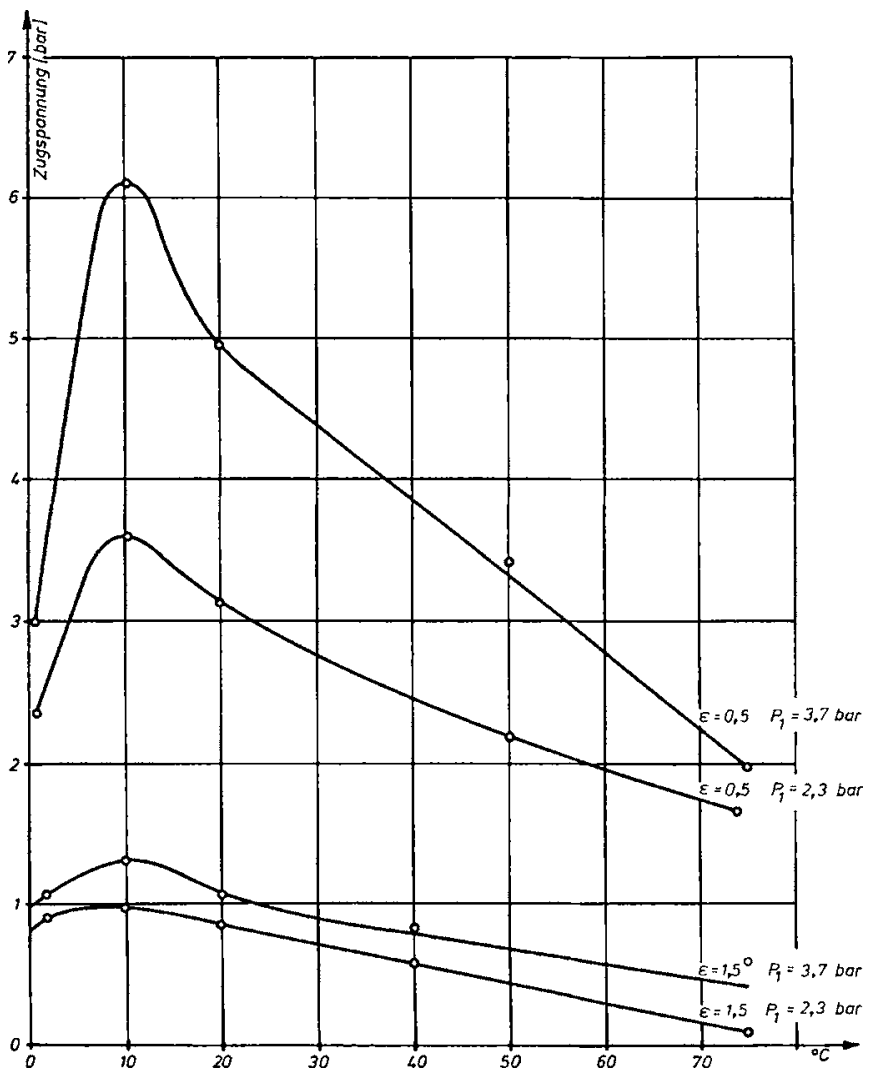

FIG. 1. Examples of the tensile strength of unfiltered tap water as functions of temperature for different degrees of gas saturation $\epsilon$ and system pressure $P_{1}$. At a given temperature, $\epsilon$ is the ratio of the actual gas content to that found at saturation at atmospheric pressure. [Courtesy of A. P. Keller (Ref. 11).]

By removing motes from distilled water by filtering, Greenspan and Tschiegg ${ }^{12}$ found that "the threshold rises as more of the motes are removed, no matter what the air content, so long as it is below saturation," i.e., the smaller the remaining motes, the higher the tensile strength. When motes larger than $0.2 \mu \mathrm{m}$ were removed, the tensile strength was raised to 100 bar for an almost unlimited time, and in excess of 200 bar for short periods in acoustic cavitation experiments carried out at $43 \mathrm{kHz}$. Filtering to even smaller mote sizes did not increase the tensile strength further. With $0.2 \mu \mathrm{m}$ motes, the gas content was found to be without importance. These measurements convincingly show that the absence of larger particles is important for achieving a high tensile strength.

In Briggs' measurements, particles present in the water may very well have been as small as $0.2 \mu \mathrm{m}$, but why did the tube wall, being itself comparable to an enormous particle, allow such high tensile strength?

Also, the insensitivity of the tensile strength to the gas content for motes of size $0.2 \mu \mathrm{m}$ or smaller, observed by Greenspan and Tschiegg, is remarkable because pronounced effects of degassing water and of pressurizing it are frequently reported in relation to lower levels of tensile strength. $4,7,13,14$ Thus, e.g., in a $46 \mathrm{kHz}$ acoustic field, Barger ${ }^{14}$ measured tensile strengths ranging from 1.2 bar in water saturated with air at 760 torr to 12.1 bar when saturated at 10 torr. Likewise, in acoustic cavitation experiments at $15 \mathrm{kHz}$ Sirotyuk $^{7}$ found that by reducing the content of surface-active substances in water until only traces of them were present, a tensile strength of 7.5 bar could be obtained. However, when the water was also degassed by evacuation, the tensile strength was raised beyond 10 bar (which was his limit of measurement). Sirotyuk also found that reduction of the conductivity of the water by two orders of magnitude did not influence its tensile strength, and his conclusion that "the ion theory fails to account for the stable existence of gas bubbles" is certainly valid. Sirotyuk did not specify to which limit he removed particles, only that "solid particles whose pores and fissures can collect air" were removed, i.e., large ones, but how large? Hereby the tensile strength of tap water/ distilled water was increased by a factor of 1.3 only, to about 1.8 bar. With Greenspan and Tschiegg's results in mind, we can assume that Sirotyuk had remaining particles notably larger than $0.2 \mu \mathrm{m}$ in his water. However, the role of remaining particles was not investigated. Sirotyuk claimed that particle "surfaces become thoroughly wetted and cannot lower the strength of the liquid appreciably," but Greenspan and Tschiegg's results as well as later studies discussed below show that this statement does not hold. It seems beyond doubt that the remaining particles were decisive for the rather limited tensile strengths he achieved. Sirotyuk also wrote, "that the stabilization of gas bubbles acting as cavitation nuclei in water is always attributable to the presence of surface-active substances." This undoubtedly holds for free gas bubbles. However, surface-active substances are undoubtedly also most relevant to gas nuclei stabilized at solid surfaces, though here they do not seem indispensable. They will certainly ease the formation of interfacial nuclei and increase their size, and they may allow such bubbles to be stable at a wide range of water-solid-vapor contact angles.

In experiments, Johnson and Cooke ${ }^{15}$ actually showed that in filtered seawater at atmospheric pressure, free gas bubbles can be stabilized by a skin. Some bubbles produced in their experiments dissolved spontaneously, and at increasing speed, due to diffusion of gas from the bubbles into the liquid. Other bubbles were stabilized by a skin within a range of diameters from $\sim 0.7$ to $13.5 \mu \mathrm{m}$, and with a maximum of their size distribution at 3-4 $\mu \mathrm{m}$. After $22 \mathrm{~h}$, the total number of stabilized bubbles was about the same, but the maximum of their size distribution had shifted to the group of smallest bubbles. At exposure to tensile stress, the bubbles expanded, and when atmospheric pressure was reestablished, they either shrank to about their original size, or they collapsed. At small pressure increase, the bubbles were slightly reduced in size; at larger increase they tended to dissolve, leaving remnants, which proved that they were indeed stabilized by a skin.

Stabilized free gas bubbles were also observed by Yount et $a l^{8}{ }^{8}$ in distilled water and in gelatine. About $5 \%$ of them were osculating. Yount developed the skin model of stabilization into the variable permeability model (VP model), according to which a skin of surface-active substances of amphiphilic nature strongly reduces or eliminates surface tension at the bubble surface. The skin is assumed to allow gas diffusion balance across the bubble surface at normal pressures, while it becomes nonpermeable at exposure to 
high pressure. From experiments, Yount estimated the skin thickness to be $2.0 \pm 0.7 \mathrm{~nm}$. In agarose gelatine, the radii of stabilized gas bubbles ranged from 0.15 to $1.35 \mu \mathrm{m}$, with the maximum number density at $0.2-0.3 \mu \mathrm{m}$. We notice a similarity with Johnson and Cooke's results for stabilized gas bubbles in seawater, though apparently in agarose the size range shifts to smaller values.

The observation of stabilized free gas bubbles of $\mu \mathrm{m}$ size in water, and their survival at exposure to (limited) compression and decompression, make them ideal candidates for cavitation nuclei, but their size distribution supports their candidature only up to moderate tensile strengths. Thus, ordinary gas bubbles of a diameter of $0.7 \mu \mathrm{m}$ at atmospheric pressure give a tensile strength of only $1.5 \mathrm{bar}$. Atchley's calculations, ${ }^{16}$ as well as subsequent calculations in the present paper, indicate that skin molecules reduce the critical pressure of the bubbles, though only to a limited extent.

When Greenspan and Tschiegg filtered their water of motes larger than $0.2 \mu \mathrm{m}$, they also removed stabilized free gas bubbles, but the remaining particles were probably contaminated to some extent by alien molecules.

The surfaces of large natural particles present in plain water do not typically have cracks and crevices, but they may be highly irregular (see $\mathrm{Crum}^{17}$ ). On this basis, the author has suggested ${ }^{18}$ that liquid detachment from concave solid surface structures causes the formation of interfacial cavitation nuclei, while convex ones obstruct their formation. This means that perfect spheres should be highly cavitationresistant, the more so the smaller they are. The hypothesis was tested and actually supported in flow experiments with a vortex nozzle by Marschall et al. ${ }^{19}$ By filtration, natural particles (and thus also stabilized free gas bubbles) larger than $1 \mu \mathrm{m}$ were removed from tap water of low gas content. The filtered water had a tensile strength of $\sim 1.3$ bar, i.e., notably smaller than the equilibrium tensile stress for vapor bubbles of $1 \mu \mathrm{m}$ diameter. With their pressure of $4 \mathrm{bar}$ in the water reservoir, we cannot attribute the measured tensile strength to skin-stabilized free gas bubbles [Eqs. (7) and (8)], but the remaining contaminated natural particles are likely candidates. Subsequently, Marschall et al. seeded the filtered water with almost spherical particles with very smooth surfaces of diameters from $\sim 3$ to $76 \mu \mathrm{m}$, Fig. 2 . When the smallest particles were seeded into the filtered water, its tensile strength was not at all influenced and remained determined by the natural motes at least three times smaller. However, when seeded with the larger particles, the tensile strength was reduced (reaching values down to $\sim 0.5$ bar for the $76 \mu \mathrm{m}$ spherical particles), but only to values much higher than the equilibrium tensile stress for vapor bubbles of size equivalent to that of the particles. Thus, the size of the critical cavities was only a small fraction of the size of the particles themselves. Consequently, in all cases smooth globally spherical particles were found to be much less inclined to cause cavitation inception than natural ones of the same size. It was conjectured by Marschall et al. that the tensile strength measured resulted from the merging of neighboring subcritical nano-voids, located at the slightly wavy surface structures of the particles, Fig. 2. In later experiments by Arora et al. ${ }^{20}$ particles from the same batches were seeded

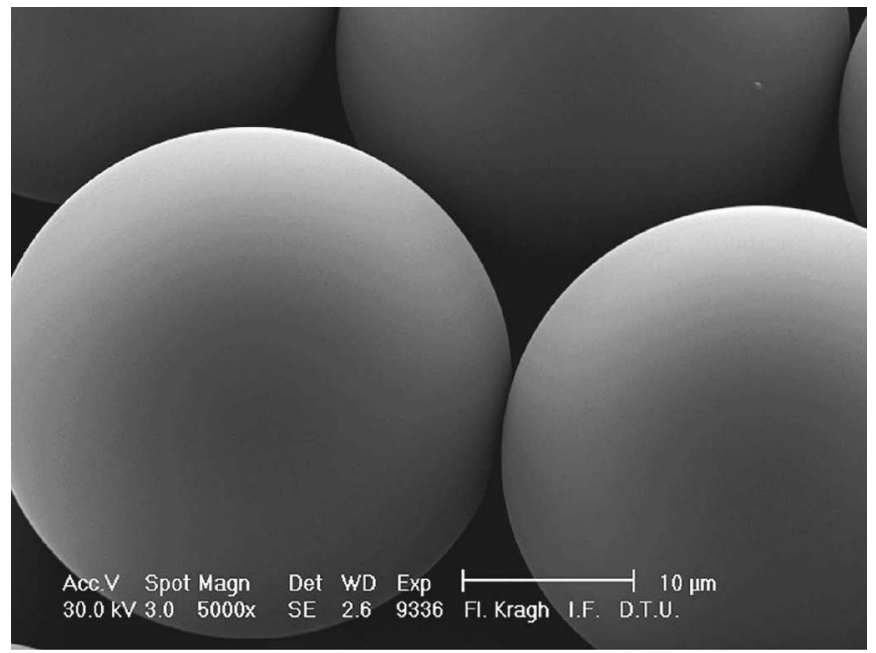

(a)

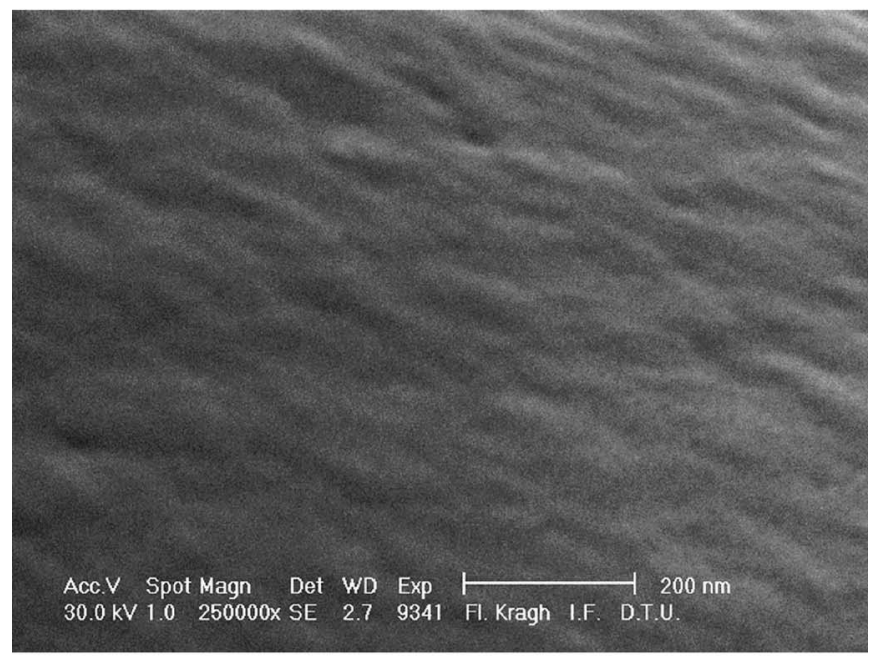

(b)

FIG. 2. Hydrophilic polystyrene particles with hydroxyl groups on their surfaces. Particle diameter $30 \mu \mathrm{m}$. Scale bar: (a) $10 \mu \mathrm{m}$, (b) $200 \mathrm{~nm}$ (Ref. 19).

into filtered and degassed water (Milli-Q water) and exposed to the transient tensile stress pulse from a lithotripter of peak amplitude 70 bar and pulse duration $<10 \mu$ s. Here cavitation inception could not be achieved. This supports the assumption that in the nozzle flow used by Marschall et al., the less clean water and the much longer time of exposure to tensile stress made it possible for the nano-voids to merge, thus causing a relatively low tensile strength.

When Arora et al. seeded almost spherical particles with corrugated surface structures, of mixed global diameters in the range 30-150 $\mu \mathrm{m}$, Fig. 3, into Milli-Q water, cavitation started when the peak of the tensile stress pulse of the lithotripter was $\sim 30$ bar. This corresponds to the formation of critical vapor bubbles of radius $\sim 50 \mathrm{~nm}$. Apparently cavitation nuclei, located at the corrugated surface structures, here reached critical size without merging with neighboring nuclei, probably because the time for such a process was insufficient. When beyond critical size, the cavities grew explosively until they were much larger than the particles 


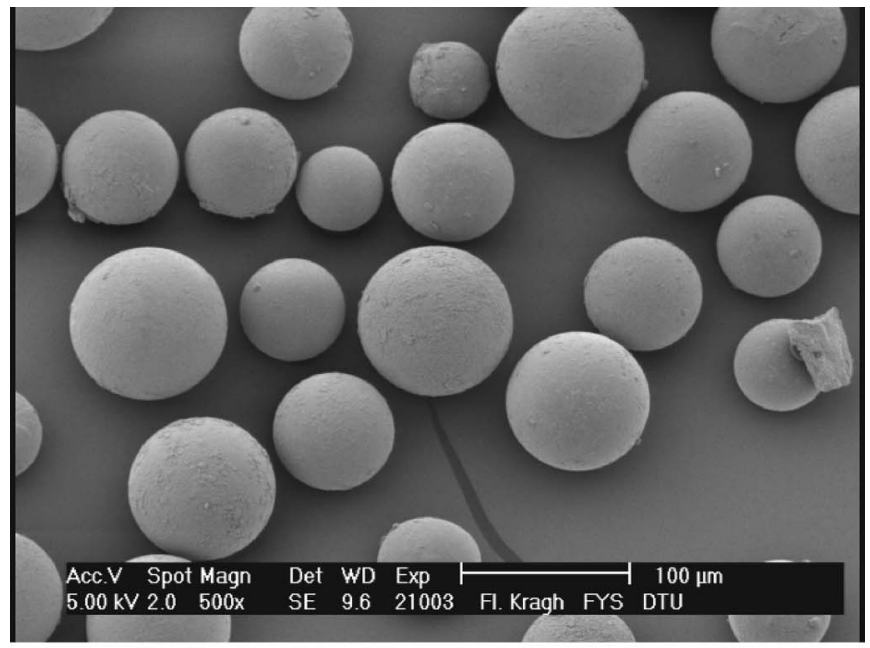

(a)

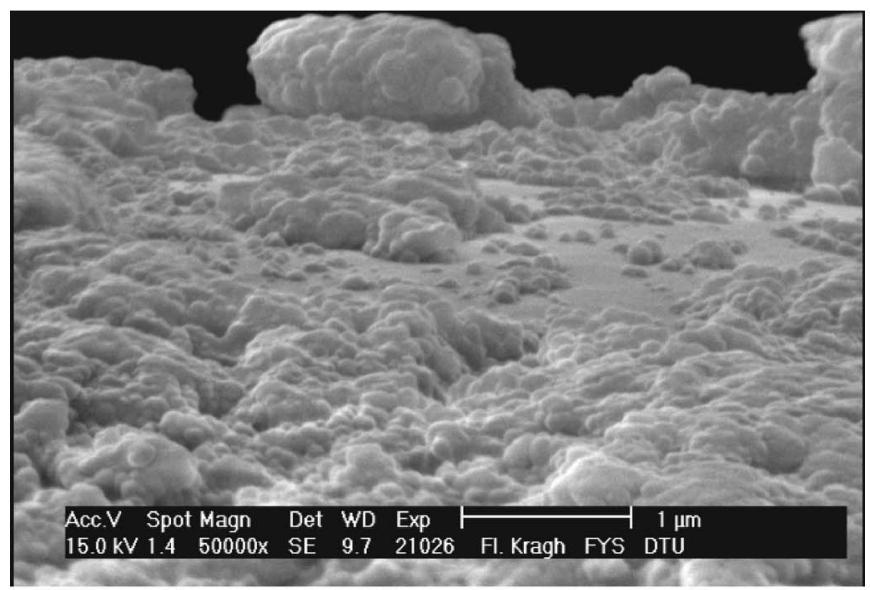

(b)

FIG. 3. Polystyrene particles with corrugated surfaces. Particle diameters 30-150 $\mu \mathrm{m}$. Scale bar: (a) $100 \mu \mathrm{m}$, (b) $1 \mu \mathrm{m}$ (Ref. 20).

nucleating them, and the particles were shot away from the cavities at high speed $(\sim 10 \mathrm{~m} / \mathrm{s})$.

Even when efforts are made to remove surface-active substances from water, remnants will be located on the surface of particles present in the liquid, and water-particle bonding is replaced by the weaker bonding of the surfaceactive substances to the particles. Thus, concave locations on the surface of particles can be expected to set up "weak points," the more so the less the water is cleaned up. In plain water containing particles of sizes up to many micrometers, and with highly irregular surfaces (cf. Ref. 17), we can imagine that neighboring voids on a particle surface merge over time into gaseous, attached microbubbles, possibly covered with surface-active substances, and reach dimensions comparable with the particles. For such water, a very small tensile strength is expected. Interfacial nano-voids have actually been observed first by scanning tunneling microscopy on surfaces of $\mathrm{W}$ and TiN submerged in distilled water, ${ }^{21}$ and later also by atomic force microscopy, e.g., on Au specimens submerged in Milli-Q water. ${ }^{22}$ By removing large natural particles from water, the maximum size of gaseous bubbles attached to the remaining particles is limited, and degassing of the water makes such gas bubbles shrink. Likewise, reduction of the content of surface-active substances reduces the size and number of skin detachment sites. Thus, the tensile strength is increased by these measures. Also, the smaller the particle size, the smaller is the global radius of a particle, and neighboring nano-voids on its surface find it increasingly difficult to merge, which raises the tensile strength.

Very small particles, even natural ones, tend to have smooth surfaces, and if concave elements occur, they are extremely small and shallow and in general separated by highly convex zones. Therefore, voids formed by liquid detachment are extremely small, and have difficulties in merging. This may explain the very high tensile strength, its lack of dependency on particle size (when smaller than $0.2 \mu \mathrm{m}$ ), as well as the lack of dependency on the gas content observed by Greenspan and Tschiegg. ${ }^{12}$ The acoustic field they used to generate tensile stress has probably caused rectified diffusion of gas into small nano-voids, and this has made them grow over time, and has limited the duration of the high tensile strength. Significant contamination of the particle surfaces with amphiphilic surface-active substances is probably required for the tensile strength to reduce to that of skin-covered vapor bubbles of equivalent size.

The very high tensile strength ( 277 bar) measured by Briggs ${ }^{8}$ in experiments at temperatures around $10{ }^{\circ} \mathrm{C}$ may be explained by the remaining natural particles being small, and above all, by Briggs' extreme cleaning of the liquid and the tube wall, making solid surfaces almost devoid of surfaceactive molecules. Apparently, the clean tube wall, being very smooth and globally only slightly concave from a molecular point of view, has been able to resist solid-liquid detachment, except at low temperatures. At temperatures approaching the freezing point, the number of solid-like layers of water molecules adjacent to the tube wall is expected to increase beyond the layer directly in contact with the solid. In that case, a concave surface curvature sets up tensile stress in the innermost layers of solid-like water, in particular in the contact layer itself, and strains its bonding to the solid surface, thus facilitating detachment. ${ }^{18}$ This applies also when natural particles of considerable surface roughness, present in ordinary tap water, are exposed to tensile stress at low temperature, which explains the observed dependency of the tensile strength of such water on the temperature. ${ }^{11}$

Johnson and Cooke's experiments, ${ }^{15}$ and Yount's, ${ }^{8}$ show that gas bubbles stabilized by a skin exist in ordinary water, but usually particles covered by such skin are also present. Let us consider the critical size of both kinds of cavitation nuclei.

\section{CRITICAL SIZE OF FREE GAS BUBBLES WITH A SKIN}

Atchley calculated the critical radius of free gas bubbles stabilized by a skin of equally charged surfactant molecules, and stabilized by a skin of polar molecules, uniformly ordered with their dipole axes in the radial direction and forming two oppositely charged concentric shells. ${ }^{16}$ However, Sirotyuk's experiments ${ }^{7}$ show that ions are not responsible for the tensile strength of water, and therefore the former 


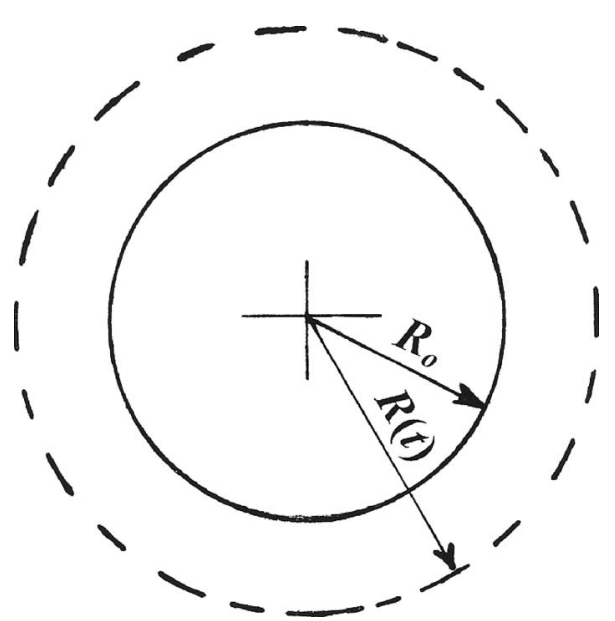

FIG. 4. A free gas bubble of radius $R_{o}$ which is fully covered by a monolayer of skin molecules with the surface tension $\gamma_{\text {skin }}=0$. This bubble is in gas diffusion balance at the far-field pressure $p_{\infty, o}$. When it is suddenly exposed to a reduced far-field pressure $p_{\infty}(t)$, it expands, and the skin breaks up into islands separated by water of surface tension $\gamma_{\mathrm{H} 2 \mathrm{O}}$. The twocomponent surface gives the bubble an effective surface tension $\gamma_{\text {eff }}$ that depends on its radius $R(t)$, Eq. (2).

configuration is of secondary interest. Likewise it is difficult to see why polar surfactants should arrange themselves as proposed in Atchley's second configuration. Yount's approach, ${ }^{8,9}$ in which VP molecules eliminate the surface tension of free gas bubbles and hereby stabilize them, seems a much more realistic choice.

Therefore, we consider here a free gas bubble in water, which shrinks by diffusion of gas until it is covered by a monolayer of amphiphilic surface-active molecules (VP molecules), whose hydrophilic heads neutralize the dangling bonds of the water molecules at the bubble surface, Fig. 4. Their hydrophobic ends form the inner surface of the stabilized bubble and set up the surface tension $\gamma_{\text {skin }}=0$. At exposure to tensile stress, the bubble expands and acquires a twocomponent surface, made up by surfactant molecules, identical in area with the stabilized bubble surface, and by water molecules, whose dangling bonds cause the surface tension $\gamma_{\mathrm{H} 2 \mathrm{O}}$. The effective surface tension of the bubble becomes a variable quantity, approaching that of a free gas bubble without a skin as its radius increases.

Such a gas bubble of initial equilibrium radius $R_{o}$ is assumed to allow gas diffusion balance with the surrounding water. The water is taken to be saturated with atmospheric air at the far field pressure $p_{\infty, o}$, and at time $t \leqslant 0$ the initial gas pressure in the bubble is

$$
p_{g, o}=p_{\infty, o}-p_{v} .
$$

Here $p_{v}$ is the vapor pressure. At $t>0$, the far-field pressure drops to $p_{\infty}(t)$, which makes the bubble expand isothermally, and areas of water form between areas of skin molecules. The total surface energy of the expanded bubble of radius $R(t)$ now defines the effective surface tension

$$
\gamma_{\mathrm{eff}}=\left\{1-\left[R_{O} / R(t)\right]^{2}\right\} \gamma_{\mathrm{H} 2 \mathrm{O}} .
$$

The gas in the bubble expands isothermally, and if diffusion is neglected, its pressure becomes

$$
p_{g}(t)=\left(p_{\infty, o}-p_{v}\right)\left[R_{o} / R(t)\right]^{3} .
$$

The excess pressure $\Delta p$ in the bubble is balanced by the effective surface tension force until critical conditions are reached, and

$$
\Delta p=p_{g}(t)+p_{v}-p_{\infty}(t)=2 \gamma_{\mathrm{eff}} / R(t) .
$$

Actually, the expanded bubble is not a perfect sphere as the areas of water have a local radius of curvature $R_{\mathrm{H} 2 \mathrm{O}}$ $=2 \gamma_{\mathrm{H} 2 \mathrm{O}} / \Delta p$, i.e.,

$$
\gamma_{\mathrm{eff}} / R(t)=\gamma_{\mathrm{H} 2 \mathrm{O}} / R_{\mathrm{H} 2 \mathrm{O}}
$$

With Eq. (2) we get

$$
R_{\mathrm{H} 2 \mathrm{O}}=R(t) /\left\{1-\left[R_{o} / R(t)\right]^{2}\right\}
$$

and

$$
\Delta p=2 \gamma_{\mathrm{H} 2 \mathrm{O}}\left\{1-\left[R_{o} / R(t)\right]^{2}\right\} / R(t) .
$$

At the critical condition $\mathrm{d}\left[p_{\infty}(t)-p_{v}\right] / \mathrm{d} R(t)=0$, or by Eq. (4), $\mathrm{d}\left[p_{g}(t)-\Delta p\right] / \mathrm{d} R(t)=0$, which gives

$$
\left(R_{\text {crit }} / R_{o}\right)^{2}=3\left[1+1 / 2\left(p_{\infty, o}-p_{v}\right) R_{o} / \gamma_{\mathrm{H} 2 \mathrm{O}}\right] .
$$

Equation (7) is identical with the equation governing gas bubbles without a skin, and in combination with Eqs. (3) and (4) it gives the usual relationship between critical pressure $p_{\infty, \text { crit }}$ and critical bubble radius $R_{\text {crit }}$,

$$
p_{v}-p_{\infty, \text { crit }}=4 \gamma_{\mathrm{H} 2 \mathrm{O}} /\left(3 R_{\text {crit }}\right) \text {. }
$$

Thus, when diffusion balance is allowed by the skin at the far-field equilibrium pressure $p_{\infty, o}$, the bubble acts precisely as an ordinary gas bubble with the same gas content, but the ordinary bubble has of course a smaller initial radius, a higher initial gas pressure, and it is not in diffusion balance.

Johnson and Cooke ${ }^{15}$ observed that at atmospheric conditions, gas bubbles in seawater could be stabilized by a skin within the range of radii $\sim 0.35 \leqslant R_{o} \leqslant 6.75 \mu \mathrm{m}$. These nuclei have critical radii of $0.67 \leqslant R_{\text {crit }} \leqslant 27 \mu \mathrm{m}$ and critical pressures of $-0.15 \mathrm{MPa} \leqslant p_{\infty, \text { crit }}-p_{v} \leqslant-3.7 \mathrm{kPa}$ [Eqs. (7) and (8)]. Johnson and Cooke observed the number density to shift over time toward the lower limit of radii, i.e., an increase of the tensile strength. Apparently the smaller size is more stable. In distilled water, Yount et al. ${ }^{8}$ observed stable osculating binaries of a radius of $\sim 1.5 \mu \mathrm{m}$. Such a binary might result from instability of a larger bubble.

If the skin is composed of amphiphilic molecules with hydrophilic heads that are wider than their hydrophobic tails, a bubble with skin molecules densely packed at both ends has a minimum of surface energy, and the skin molecules define a preferential bubble size. A smaller bubble diameter would make the hydrophilic heads detach from each other, leaving dangling bonds of water molecules between them, i.e., the potential energy of the system would increase. In contrast, a larger bubble diameter would make the inner hydrophobic tails separate. It would cost little work only, but would make the structure less stable, and it might break up.

The calculated range of critical pressures for observed skin-stabilized free gas bubbles in water at atmospheric pressure shows that such bubbles are not responsible for the high tensile strength measured when water is thoroughly 


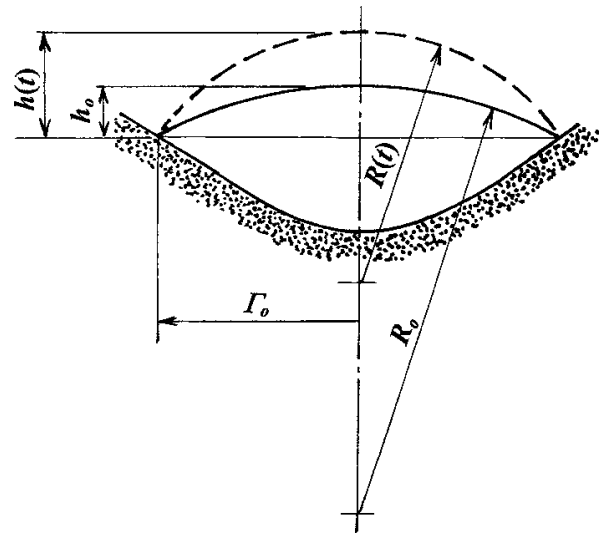

FIG. 5. Solid surface submerged in water that has detached from a concave element, thus forming an interfacial void. The void surface is shown at initial conditions $\left(R_{o}\right)$, and during expansion $[R(t)]$ driven by a reduction of the far-field pressure.

cleaned $^{7,10}$ or just distilled, and motes larger than $0.2 \mu \mathrm{m}$ are removed. ${ }^{12}$ However, it is well known that clean surfaces contaminate very quickly when exposed to the environment, and generally surface-active molecules can be expected to form a skin around particles in water.

\section{PARTICLES AND BOUNDING WALLS WITH A SKIN}

If particles in water, or walls bounding it, are covered by a monolayer of surface-active molecules, the adhesion of the layer is determined by the weak van der Waal's forces, caused by the hydrophobic tails of the molecules, while their hydrophilic heads bond strongly to the adjacent water molecules. At concave surface structures the layer may detach, allowing the formation of a void into which gas molecules diffuse until equilibrium is achieved.

Let us consider such a concave location with a skin that has detached and forms an axisymmetric spherical cap with a radius of curvature $R_{o}$, with attachment radius $\Gamma_{o}$ and height $h_{o}$, Fig. 5. The skin has the surface tension $\gamma_{\text {skin }}=0$. The volume of the void $V_{o}$ consists of the volume of the spherical segment $V_{\mathrm{sph}, o}$ and the subvolume $V_{s}$ beneath it, governed by the surface geometry,

$$
\begin{aligned}
V_{o} & =V_{s}+V_{\mathrm{sph}, o} \\
& =V_{s}+\pi h_{o}\left(3 \Gamma_{o}^{2}+h_{o}^{2}\right) / 6=V_{s}+\pi h_{o}^{2}\left(R_{o}-h_{o} / 3\right),
\end{aligned}
$$

where $R_{o}=\left(\Gamma_{o}^{2}+h_{o}^{2}\right) /\left(2 h_{o}\right)$.

We assume that for time $t \leqslant 0$ the gas in the void is in diffusion balance at the saturation pressure $p_{g, o}=p_{\infty, o}-p_{v}$. At $t=0$, the far-field pressure drops to $p_{\infty,}(t)<p_{\infty, o}$, and the spherical cap with the initial area $A_{o}$ expands into a twocomponent cap with an area $A(t)$, composed of skin as well as of water elements. $\Gamma_{o}$ remains constant, because a change of the contact radius is a slow process in which molecular bonds at the liquid-solid interface break successively, and this takes time. Thus, $R_{o} \rightarrow R(t), h_{o} \rightarrow h(t)$, and $V_{o} \rightarrow V(t)$, and the gas pressure becomes

$$
p_{g}(t)=p_{g, o} V_{o} / V(t) .
$$

The spherical cap of area $A(t)=2 \pi R(t) h(t)=\pi\left[\Gamma_{o}^{2}+h(t)^{2}\right]$ acquires an effective surface tension

$$
\gamma_{\mathrm{eff}}=\left[h(t)^{2}-h_{o}^{2}\right] \gamma_{\mathrm{H} 2 \mathrm{O}} /\left[\Gamma_{o}^{2}+h(t)^{2}\right] .
$$

The force balance, Eq. (4), requires

$$
p_{v}+p_{g}(t)-p_{\infty}(t)=4 \gamma_{\mathrm{H} 2 \mathrm{O}} h(t)\left[h(t)^{2}-h_{o}^{2}\right] /\left[\Gamma_{o}^{2}+h(t)^{2}\right]^{2} .
$$

The void volume $V(t)$ grows with $h(t)$, and critical conditions are achieved when

$$
\begin{aligned}
\mathrm{d}\left[p_{\infty}(t)-p_{v}\right] / \mathrm{d} h(t)= & \mathrm{d}\left[p_{g}(t)\right] / \mathrm{d} h(t)-4 \gamma_{\mathrm{H} 2 \mathrm{O}}\left\{\Gamma_{o}^{2}\left[3 h(t)^{2}-h_{o}^{2}\right]\right. \\
& \left.-h(t)^{2}\left[h(t)^{2}-3 h_{o}^{2}\right]\right\} /\left[\Gamma_{o}^{2}+h(t)^{2}\right]^{3}=0 .
\end{aligned}
$$

In the extreme case of a void with a flat detached skin, $h_{\mathrm{o}}$ $=0$, and if the solid surface is very smooth so that $V_{s}$ is very small, the gas content becomes negligible at void expansion, even though $p_{g, o}=1$ bar at atmospheric far-field conditions when $t \leqslant 0$. Then Eq. (13) gives $h_{\text {crit }}=\sqrt{3} \Gamma_{o}$, which corresponds to $R_{\text {crit }}=2 \Gamma_{o} / \sqrt{3}$ and a critical tensile strength $-\left(p_{\infty, \text { crit }}-p_{v}\right)=3 \sqrt{3} \gamma_{\mathrm{H} 2 \mathrm{O}} /\left(4 \Gamma_{o}\right)$. We notice that $\Gamma_{o}$ has no lower limit (except one related to the atomic structure of the solid surface), and at a decrease of $\Gamma_{o}$ the tensile strength rises correspondingly. Without the skin, such a void becomes critical when it is hemispherical, $R_{\text {crit }}=\Gamma_{o}$ and $p_{\infty, \text { crit }}$ $=-2 \gamma_{\mathrm{H} 2 \mathrm{O}} / \Gamma_{o}$. Thus, the skin makes the critical void radius slightly larger, and it reduces the critical tensile strength moderately.

If the solid surface is corrugated and detachment of the skin occurs, then $V_{s}>0$, and maybe also $h_{o} \geqslant 0$. (Actually, $h_{o}$ may grow over time due to surfactant molecules being picked up.) For such cases, $\mathrm{d} p_{g}(t) / \mathrm{d} h(t)<0$ when pressure drop occurs in the far field, which leads to an increase of $R_{\text {crit }}$ and a decrease of the tensile strength $-\left(p_{\infty, \text { crit }}-p_{v}\right)$ relative to the values calculated above for $V_{s} \approx 0$ and $h_{o}=0$.

In accordance with experimental experience, this model satisfies that extremely clean and smooth solid surfaces immersed in highly cleaned water result in very small cavitation nuclei and in a high tensile strength, in particular if the surfaces are convex. In Briggs' experiments, ${ }^{10}$ the cleanliness of the water, a very small size of remaining particles, and the smoothness of the clean glass tube used, though globally concave, explain the very high tensile strength, which he measured at $\sim 10^{\circ} \mathrm{C}$. The globally concave curvature of the tube actually offers an explanation of the sharp drop of tensile strength, because when the temperature approaches $0{ }^{\circ} \mathrm{C}$ the number of solid-like layers of water molecules adjacent to the solid surface increases, and it strains the water-solid bonding. Likewise, the model explains the high tensile strength obtained by Greenspan and Tschiegg when only natural particles smaller than $0.2 \mu \mathrm{m}$ (Ref. 12) were left in the water, and the lack of sensitivity to the gas content for such water. Contrary to very small natural particles, larger ones are in general of irregular shape, and this leads to sensitivity to the gas content and to a much lower tensile strength. This offers an interpretation of Sirotyuk's results, ${ }^{7}$ 
in which the tensile strength exceeded 10 bar in only a single case. The measurements of Marschall et al. ${ }^{19}$ and Arora et $a l .{ }^{20}$ carried out with artificial, almost perfectly spherical, smooth $30 \mu \mathrm{m}$ particles from the same batch, are illustrating. When seeding these particles into tap water filtered of natural particles larger than $1 \mu \mathrm{m}$, Marschall et al. obtained a tensile strength of $\sim 0.9$ bar in a nozzle flow of filtered tap water. When particles from the same batch were seeded into Milli-Q water and exposed to a lithotripter shock wave by Arora et al., they did not cavitate even at 70 bar tensile stress. This points to both the importance of the cleanliness of the water, as stressed by Briggs as well as by Sirotyuk, and to an influence of the time of exposure to tensile stress. However, it should also be noted that the lithotripter wave is an $\mathrm{N}$-wave with a compressive stress pulse at least as strong as the subsequent tensile stress pulse. The compressive phase may have eliminated temporarily the very flat interfacial voids, or at least reduced them significantly. In Arora et al.'s further experiments with spherical particles that had a rough surface, and were of diameters in the 30-150 $\mu \mathrm{m}$ range, the tensile strength was $\sim 30 \mathrm{bar}$, and cavitation occurred on particles of all sizes. This indicates that larger nuclei were harbored in the rough surface structures, but merging of individual voids did not have time to develop. In Marschall et al.'s flow experiments, the compressive pulse was absent, and the tensile stress was low, but its duration was about two orders longer, allowing merging of nano-voids to occur, and it was facilitated when the global curvature of the particles was reduced, i.e., at increase of the particle size.

Keller's measurements of a decrease of the tensile strength of water in flow channels with unfiltered tap water when the temperature dropped from $10{ }^{\circ} \mathrm{C}$ toward zero ${ }^{11}$ also point to particles with irregular surfaces ${ }^{18}$ being the general source of cavitation nuclei in such systems. The convex surfaces of free gas bubbles stabilized by a skin cannot explain this temperature dependency. However, it would be most interesting to see measurements of the temperature dependency of the tensile strength of seawater containing free gas bubbles stabilized by a skin, covering the range of temperatures from 15 to $\sim 0{ }^{\circ} \mathrm{C}$.

It is worthwhile to recall Hannes Alfvén's words: "Theories come and go- the experiment is here forever." In cavitation research, we already have a huge amount of experimental evidence. These measurements and the experimental conditions at which they are obtained, known as well as un- known, reflect nature itself and offer us the possibility of building models that satisfy the sum of experimental knowledge. From such models we can plan new experiments that give us an even deeper insight. It is a challenge to be met.

${ }^{1}$ J. C. Fisher, "The fracture of liquids," J. Appl. Phys. 19, 1063 (1948).

${ }^{2}$ Q. Zheng, D. J. Durben, G. H. Wolf, and C. A. Angell, "Liquids at large negative pressures: Water at the homogeneous nucleation limit," Science 254, 829 (1991).

${ }^{3}$ P. S. Epstein and M. S. Plesset, "On the stability of gas bubbles in liquidgas solutions," J. Chem. Phys. 18, 1505 (1950).

${ }^{4}$ E. N. Harvey, D. K. Barnes, W. D. McElroy, A. H. Whiteley, D. C. Peace, and K. W. Cooper, "Bubble formation in animals," J. Cell. Comp. Physiol. 24, 1 (1944).

${ }^{5}$ F. E. Fox and K. F. Herzfeld, "Gas bubbles with organic skin as cavitation nuclei," J. Acoust. Soc. Am. 26, 984 (1970).

${ }^{6} \mathrm{~V}$. A. Akulichev, "Hydration of ions and the cavitation resistance of water," Sov. Phys. Acoust. 12, 144 (1966).

${ }^{7}$ M. G. Sirotyuk, "Stabilization of gas bubbles in water," Sov. Phys. Acoust. 16, 237 (1970).

${ }^{8}$ D. E. Yount, E. W. Gillary, and D. C. Hoffman, "A microscopic investigation of bubble formation nuclei," J. Acoust. Soc. Am. 76, 1511 (1984).

${ }^{9}$ D. E. Yount, "On the elastic properties of the interfaces that stabilize gas cavitation nuclei," J. Colloid Interface Sci. 193, 50 (1997).

${ }^{10}$ L. J. Briggs, "Limiting negative pressure in water," J. Appl. Phys. 21, 721 (1950).

${ }^{11}$ A. P. Keller, "Schlussbericht über Das Forschungsvorhaben," Beginnende Kavitation, Zugspannungen in Flüssigkeiten, Oskar von Miller-Institut, Technischen Universität München/Obernach, Germany (1982), 2. Teil, p. 15.

${ }^{12}$ M. Greenspan and C. E. Tschiegg, "Radiation-induced acoustic cavitation: Apparatus and some results," J. Res. Natl. Bur. Stand., Sect. C 71C, 299 (1967).

${ }^{13}$ M. Strasberg, "Onset of ultrasonic cavitation in tap water," J. Acoust. Soc. Am. 31, 163 (1959).

${ }^{14}$ J. E. Barger, "Thresholds of acoustic cavitation," Technical Memorandum No. 57, Acoustics Research Laboratory, Harvard University, ONR Contract 1866(24) (1964)

${ }^{15}$ B. D. Johnson and R. C. Cooke, "Generation of stabilized microbubbles in seawater," Science 213, 209 (1981).

${ }^{16}$ A. A. Atchley, "The Blake threshold of a cavitation nucleus having a radius-dependent surface tension," J. Acoust. Soc. Am. 85, 152 (1989).

${ }^{17}$ L. A. Crum, "Tensile strength of water," Nature 278, 148 (1979).

${ }^{18} \mathrm{~K}$. A. Mørch, "Cavitation nuclei and bubble formation-A dynamic liquid-solid interface problem," J. Fluids Eng. 122, 494 (2000).

${ }^{19}$ H. B. Marschall, K. A. Mørch, A. P. Keller, and M. Kjeldsen, "Cavitation inception by almost spherical solid particles in water," Phys. Fluids 15, 545 (2003).

${ }^{20}$ M. Arora, C.-D. Ohl, and K. A. Mørch, "Cavitation inception on microparticles: A self-propelled particle accelerator," Phys. Rev. Lett. 92, 174501 (2004)

${ }^{21}$ J. P. Song, K. A. Mørch, K. Carneiro, and A. R. Thölén, "STM investigations of solid surfaces in water and air," Surf. Sci. 296, 299 (1993).

${ }^{22}$ M. Holmberg, A. Kühle, J. Garnæs, K. A. Mørch, and A. Boisen, "Nanobubble trouble on gold surfaces," Langmuir 19, 10510 (2003). 